\title{
Stability and Convergence of TCP-like Congestion Controllers in a Many-Flows Regime
}

\author{
Supratim Deb \\ Coordinated Science Lab, \\ University of Illinois, \\ Urbana, Illinois, USA \\ Email: deb@uiuc.edu
}

\author{
Sanjay Shakkottai \\ Department of Electrical and \\ Computer Engineering \\ University of Texas, Austin, USA \\ Email:shakkott@ece.utexas.edu
}

\author{
R. Srikant \\ Coordinated Science Lab, \\ University of Illinois, \\ Urbana, Illinois, USA \\ Email: rsrikant@uiuc.edu
}

\begin{abstract}
With the rapid growth of Internet, parameter design and analysis for large-scale networks has become a topic of active interest. Since simulation of such large scale systems is not easy, deterministic fluid models have been widely used for both qualitative understanding of the behavior, as well as parameter design for such networks.

In this paper, we first study a deterministic fluid model for Internet congestion control when there are multiple TCP-like flows present. We provide conditions under which such a system is globally asymptotically stable in the presence of feedback delay.

We then study the corresponding system with the addition of web mice and other non-responsive flows modeled as stochastic disturbances. We show that, when there are a large number of flows, choosing parameters based on the global stability criterion for the deterministic system (with the noise replaced by its mean value) ensures global stability for the stochastic system as well.

Numerical examples and simulation results with some popular active queue management mechanisms validate the parameter choices from analysis. The results indicate that a system with multiple TCP-like flows is globally stable as long as the bandwidthdelay product per flow is not very small.
\end{abstract}

\section{INTRODUCTION}

The design philosophy of the current Internet is based on the end-to-end paradigm, wherein most of the intelligence is at the end hosts. The network's task is to simply notify the end systems whenever it detects congestion in the network. Congestion detection is based on the aggregate flow behavior at the router, and the end-hosts are notified by simply dropping or by marking packets using the explicit congestion notification (ECN) bit [4]. The end-host reacts to this information by decreasing its transmission rate, thus adapting to network congestion. In this manner, end-to-end control is maintained using only minimal network resources.

This end-to-end design philosophy has motivated a lot of work [7], [10] using a utility function maximization framework, leading to a class of end-to-end rate control mechanisms for Internet congestion control. Based on the choice of the utility function, various types of fairness amongst users can be achieved. Further, it has been shown in [10] that the congestion-avoidance phase of TCP flow-control can be considered a special case of the above framework for appropriately chosen utility functions. Such deterministic rate based models of TCP have led to better understanding of TCP and points the way to improve existing congestion control mechanism used in the Internet.

Deterministic rate update models which explicitly account for round-trip delay have been the focus of much study in the recent past. An important question that has been addressed deals with stability of network controllers based on these deterministic rate adaptation mechanisms. In [8], a stability condition for single proportionally fair congestion controller with delayed feedback was provided. Since then, this result has been extended to networks in [6], [12], [17], and in [13], similar results were shown for a different class of controllers. We also refer the reader to [5], [11] for other related analysis of congestion controllers with delay. All of the above work dealt with local stability of the linearized controllers in the presence of round-trip delay. More recently, sufficient conditions were derived in [3] for global exponential stability for the case of a single flow accessing a link.

However, one may ask why we should consider deterministic models for Internet congestion control. In realistic systems, there are two sources of randomness. First, there can be flows that do not react to congestion control. For instance, these could be in the form of web-mice, which are short flows which terminate before they can react to congestion control. Such uncontrolled flows can be modeled as stochastic disturbances at the router.

Second, the marking decisions at the router could be probabilistic. To see this, consider a particular time-instance where the router decides to mark $20 \%$ of the packets. Due to the constraint that the router can toggle the state of only a single bit in the packet header, a possible strategy is to mark each packet independently, and with probability 0.2 .

In this paper we concern ourselves with randomness generated due to uncontrolled short flows, in a many-flows regime. We refer the reader to [2] for an analysis of TCP behavior in the context of probabilistic marking at the router.

In [16], the authors justified using fluid deterministic model for studying proportionally fair congestion controllers. They showed that, in the many-flows regime, the trajectory of the average rate at the router converged to that of the deterministic model with the noise replaced by its mean. 


\section{A. Main Contribution}

In this paper, we consider a system consisting of a single link accessed by a large number of TCP-like flows, each with identical feedback delay, but with (possibly) different initial conditions and also accessed by a large number of uncontrolled flows. By a TCP-like mechanism, we refer to the rate control model of the congestion avoidance phase of TCP proposed in [10], which is also closely related to the model in [5]. We are interested in relating this stochastic model to a deterministic model where the noise process is replaced by its mean.

We show that, in the presence of uncontrolled flows modeled as stochastic noise, the deterministic fluid model with noise replaced by its mean value is accurate in the following sense: The average rate of the flows behaves like a single flow asymptotically in the number of flows and time. Thus, unlike in the proportionally-fair case studied in [16] where convergence was shown for each time (as opposed to asymptotically in time), here, the trajectory of the stochastic system does not converge to that of the deterministic system in the many-flows regime.

However, if the number of flows is large enough, the global stability criterion for a single flow (with minor modifications) is also a global stability condition for the stochastic system with multiple flows. Thus, the implication is that parameter design can be carried out using deterministic analysis based on the single flow model.

Further, for some standard marking functions used in literature, we show that TCP-like sources with standard TCP parameters satisfy the stability criterion when the bandwidthdelay product per source is sufficiently large.

\section{B. Organization of this Paper}

We begin with a description of the model in Section II. We consider multiple TCP-like flows along with uncontrolled flows in the model. In Section III, we study the system by simply considering the mean of the uncontrolled flow rate through the link. We present conditions on the congestion control gain for the global stability of such a system. The next natural question is whether such a choice of gain parameter is the right one when the disturbance process due to uncontrolled flows is not a constant but a time-varying (possibly stochastic) process. We answer this question in Section IV. We show that the choice of gain parameter with uncontrolled flows modeled as a constant is appropriate when the number of flows is large. In Section $\mathrm{V}$ we study the conditions derived in the context of current TCP parameters and give examples to illustrate the results. We provide some simulation results in Section VI before concluding in Section VII.

\section{SySTEM MODEL}

Our model is that of a single bottleneck link being accessed by many TCP-like flows. The delay in the forward and the reverse path is $d / 2$ so that the round-trip delay of each source is $d$. Such a model can be applicable in a scenario when multiple users behind an ISP access a server through a common bottleneck link as in Figure 1. The number of users

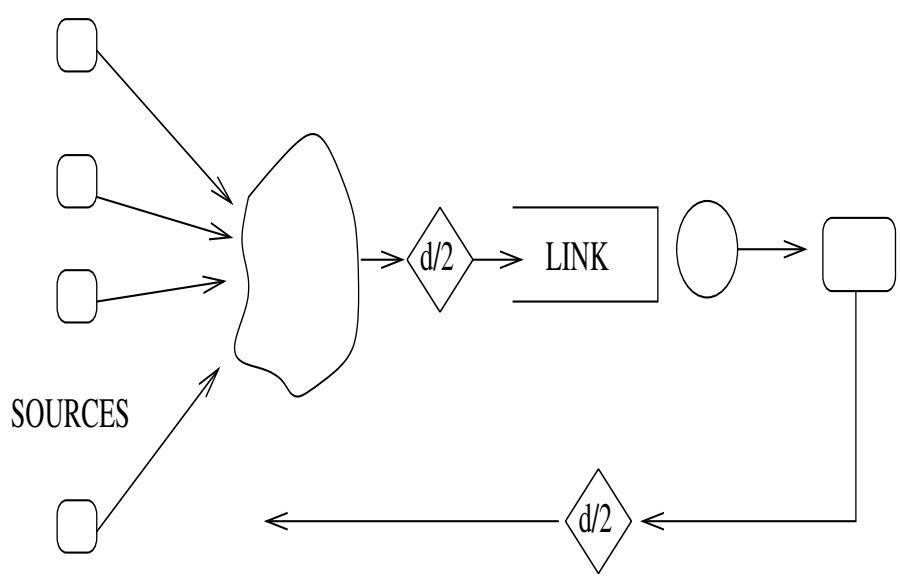

Fig. 1. The system model

in the system is $N$, which is also the scaling parameter. We consider a sequence of such systems indexed by $N$. In the $N$-th system, there are $N$ sources accessing the link and the capacity of the link is scaled as $N c$ so that capacity per source is maintained at $c$. Further, in the $N$-th system there are $N$ uncontrolled flows accessing the link. Before we describe the rate update mechanism of the sources we first comment on the marking function of the link.

The link has a marking function $p(\lambda, C)$ which denotes the fraction of packets marked when the total arrival rate into the link is $\lambda$ and the link capacity is $C$ (where $C=N c$ ). The marking function is assumed to satisfy the following conditions.

\section{Assumption 1: (Marking Function)}

1) The function $p(\lambda, C)$ is increasing in $\lambda$ and is Lipschitz continuous in $\lambda$.

2) We further assume that $p(\lambda, C)=p(\lambda / C, 1)$.

The first assumption is obvious since $p(\lambda, C)$ is the fraction of packets marked. The second assumption says that the fraction of packets marked simply depends on the ratio of the total arrival rate and the link capacity. To understand this property in the context of our scaling, suppose in the $N$-th system, the rate of the $i$-th controlled flow is $x_{i}$ and the rate of the $i$-th uncontrolled flow is $e_{i}$ for $1 \leq i \leq N$. Then the marking function for the $N$-th system is,

$$
p(\lambda, N c)=p\left(\frac{\sum_{i}\left(x_{i}+e_{i}\right)}{N c}, 1\right)=p\left(\frac{x+e}{c}, 1\right),
$$

where $x$ and $e$ are the average rate of the controlled and the uncontrolled flows respectively. Thus, under this assumption, the marking function in the $N$-th system simply depends on the average flow rate through the link for some fixed capacity per flow. Two examples of marking functions which have this property are:
1. $p(\lambda, C)=\left(\frac{\lambda}{C}\right)^{B}$
2. $p(\lambda, C)=\frac{a \lambda}{C-(1-a) \lambda}$.

The first marking function has the interpretation of the queue size being $B$ or larger in an $M / M / 1$ queue with arrival 
rate $x$. The second marking function can be used a rate based model for REM for a suitable choice of $a$ [8]. We remark that any reasonable marking function should satisfy the second assumption. This ensures the scalability of the marking function in the number of flows. Thus, from now on, we will interpret the arguments $x$ and $c$ of the marking function $p(x, c)$, as the average arrival rate and the capacity per flow respectively. Further, in the systems we consider from now on, the capacity per flow $c$ will remain constant, and the only time-varying parameter is the average rate $x$. Thus, to avoid unnecessary notation, we will hide the dependence of $p(\cdot, c)$ on $c$, and let

$$
p(x) \equiv p(x, c)=p(x / c, 1)
$$

In addition to the controlled flows, we assume that the system is accessed by uncontrolled flows. These are flows which do not react to congestion signals and are modeled as stochastic processes with mean $a$. In the $N$-th system, there are $N$ uncontrolled flows $\left\{e_{i}^{(N)}(t)+a\right\}_{i=i}^{N}$ accessing the link. We model $\left\{e_{i}^{(N)}(t)\right\}_{i=1}^{N}$ as i.i.d and bounded stochastic processes with mean 0 . We assume that $e_{i}^{(N)}(t)$ 's satisfy

$$
\lim _{N \rightarrow \infty} \sup _{t \in[0, N T)} \frac{1}{N} \sum_{i=1}^{N} e_{i}^{(N)}(t)=0 \text { a.s. . }
$$

We now describe our model for the controlled flows. We consider a fluid model for the rate update of the controlled flows. Denote by $y_{i}^{(N)}(t)$ the flow rate of the $i$-th flow at time $t$ when there are $N$ such flows present in the system. Further, denote by $x^{(N)}(t)$ the average flow rate of the controlled flows through the link at time $t$ and so

$$
x^{(N)}(t)=\frac{1}{N} \sum_{i=1}^{N} y_{i}^{(N)}(t) .
$$

Similarly, denote by $\left(e^{(N)}(t)+a\right)$ the mean flow rate of the $N$ uncontrolled flows through the link. The fraction of packets marked by the link is $p\left(x^{(N)}+a+e^{(N)}(t)\right)$, where the average flow rate at the link consists of the average flow rate of the controlled flows $x^{(N)}(t)$ and the average flow rate due to $N$ uncontrolled flows $a+e^{(N)}(t)$. The rate update of the $i$-th flow is governed by,

$$
\begin{aligned}
& \dot{y}_{i}^{(N)}(t)=\kappa[w- \\
& \left.\quad y_{i}^{(N)}(t) y_{i}^{(N)}(t-d) p\left(x^{(N)}(t-d)+a+e^{(N)}(t-d / 2)\right)\right], \\
& \quad i=1,2, \ldots, N,
\end{aligned}
$$

where $\kappa>0$ is a constant and determines stability in the presence of delay. Note that, the feedback delay is constant. This is reasonable if we employ early congestion notification schemes using virtual queues which lead to negligible queueing delays at the router. We comment that choosing $\kappa=2 / 3$ and $\kappa w=1 / d^{2}$ results in the rate control model of TCP [10]. It can be shown that, in the absence of delay and by simply taking the mean of the noise process contributed by the uncontrolled flows, the above system converges to the unique equilibrium point given by the solution of

$$
y_{i}^{*} \sqrt{p\left(y_{i}^{*}+a\right)}=\sqrt{w} .
$$

In the rest of the paper, we find conditions under which the system converges to the equilibrium point in the presence of feedback delay, with and without stochastic disturbance introduced by the uncontrolled flows. We also discuss the implication of our results in the context of TCP.

\section{Multiple TCP-LiKe Flows With IDENTICAL Round-TRip Delays And CONSTANT NoIsE}

In this section we study the system when the noise process due to the uncontrolled flows is simply modeled by a constant process of rate $a$. For the purposes of this section we keep $N$, the number of flows, fixed and so we drop the superscript on $y_{i}^{(N)}(t)$ and $x^{(N)}(t)$ and simply denote them by $y_{i}(t)$ and $x(t)$ respectively.

The rate update of the $i$-th flow is,

$$
\begin{gathered}
\dot{y}_{i}(t)=\kappa\left[w-y_{i}(t) y_{i}(t-d) p(x(t-d)+a)\right], \\
i=1,2, \ldots, N,
\end{gathered}
$$

and the trajectory of the average flow rate through the link $x(t)$ can be described by

$$
\dot{x}(t)=\kappa\left[w-\left[\frac{1}{N} \sum_{1}^{N} y_{i}(t) y_{i}(t-d)\right] p(x(t-d)+a)\right] .
$$

Our goal is to find suitable conditions on $\kappa$ for the above system to converge to its unique equilibrium point.

We first provide conditions under which the average flow rate $x(t)$ is bounded. The following result which is derived in [15] provides such a condition.

Lemma 3.1: [15] Suppose $\kappa d<\beta$. Fix $\delta>0$, where $\delta$ can be arbitrarily small. Then there exists $t_{0}(\delta)<\infty$ such that for all $t>t_{0}$

$$
x(t) \leq M_{\beta},
$$

where $M_{\beta}$ is the smallest positive number satisfying

$$
M_{\beta}^{2} p\left(M_{\beta}+a-2 w\right)\left(1-\frac{2 w \beta}{M_{\beta}}\right) \geq w+\delta
$$

For the rest of this paper, we will assume that $\delta$ in the preceding theorem is a very small number, fixed at, say, $\delta=0.0001$. Without loss of generality, we study the system evolution from time $t_{0}$ onwards. Thus (by shifting the timeaxis), throughout the paper we assume that $x(t) \leq M_{\beta}$ for all $t \geq 0$. Suppose the initial value of the average rate lies in some compact set $[0, K]$ in which the equilibrium point of the system is included. Then, clearly, there exists a $\beta$ for which $M_{\beta} \geq K$. We now assume that initial condition for each flow satisfies

Assumption 2: (Initial Condition) The initial trajectory for any user $i \in\{1,2 \ldots N\}$ satisfies

$$
M_{\beta}(1-\epsilon) \leq y_{i}(s)<M_{\beta}(1+\epsilon), \forall s \in[-d, 0]
$$


for some $\epsilon<1$.

Essentially, this says that the initial values of the individual user rates are not too far away from each other. Since the value of $M_{\beta}$ is larger than the equilibrium rate, not allowing the initial user rates to be more than twice the value of $M_{\beta}$ is a reasonable assumption.

We now introduce the following notation for every pair of flows.

$$
r_{i j}(t)=y_{i}(t)-y_{j}(t), \quad(i, j) \in\{1,2, \ldots, N\}^{2}
$$

Our goal is to show that $r_{i j}(t)$ converges to zero for appropriately chosen $\kappa$. This will enable us to show that the system indeed converges to the unique equilibrium point under suitable conditions.

First, note that the dynamics of $r_{i j}(t)$ can be described by the following.

$$
\begin{aligned}
\dot{r}_{i j}(t) & \\
= & -\kappa p(x(t-d)+a)\left[y_{i}(t) y_{i}(t-d)-y_{j}(t) y_{j}(t-d)\right] \\
= & -\kappa p(x(t-d)+a)\left[y_{i}(t) y_{i}(t-d)-y_{i}(t) y_{j}(t-d)\right. \\
& \left.+y_{i}(t) y_{j}(t-d)-y_{j}(t) y_{j}(t-d)\right] \\
= & -\kappa p(x(t-d)+a)\left[y_{i}(t) r_{i j}(t-d)+\right. \\
& \left.y_{j}(t-d) r_{i j}(t)\right]
\end{aligned}
$$

Before we state our result on the convergence of $r_{i j}(t)$ we restate the following result from [3] on general timevarying linear delay differential equations. This will be useful in proving the convergence of $r_{i j}(t)$.

Lemma 3.2: [3] Consider the delay differential equation given by

$$
\dot{x}(t)=a(t) x(t)+b(t) x(t-d)
$$

with some initial condition, $x(t)=\phi(t), t \in[-d, 0]$. If there exists $q>1$ such that $a(t)$ and $b(t)$ satisfy

$$
d \sqrt{q} \max _{t-d \leq s \leq t}(|a(s)|+|b(s)|)<-\operatorname{sgn}(b(t))-\frac{a(t)}{|b(t)|},
$$

for all $t \leq t_{1}$, then

$$
V(t)<q V(0) e^{-\alpha t}, \quad \forall t \leq t_{1},
$$

where $V(x(t))=\sup _{t-2 d \leq s \leq t} x^{2}(s)$ and $\alpha>0, q>1$ are constants.

We are now in a position to state and prove the following result on the convergence of $r_{i j}(t)$.

Theorem 3.1: If $\kappa d$ satisfies

$$
\kappa d<\min \left[\beta, \frac{1}{6 M_{\beta} p\left(M_{\beta}+a\right)}\right],
$$

then

$$
\lim _{t \rightarrow \infty} \sup _{(i, j) \in\{1,2, \ldots, N\}^{2}} r_{i j}(t)=0 .
$$

Remark: Before we go into the details of the proof we will illustrate the key idea in the proof informally. First, we can show that as long as all the flow rates $\left\{y_{i}(t)\right\}$ are less than $3 M$, the difference between their rates $\left\{r_{i j}(t)\right\}$ will decrease. This will follow from the single flow global stability condition from

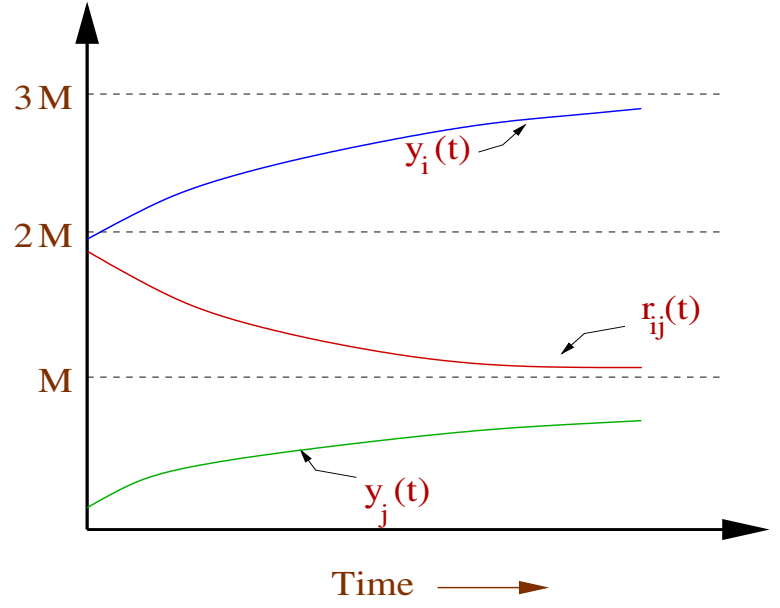

Fig. 2. Main proof idea in Theorem 3.1

Lemma 3.2. Second, recall from Lemma 3.1 that the average rate is upper-bounded as well, i.e., $x(t)<M$ (see Figure 2).

Suppose at some time, say $t_{1}$, it happens that for a particular flow $l, y_{l}\left(t_{1}\right)=3 M$, and up to time $t_{1}$, we have all the individual flow rates strictly less than $3 M$. As the average rate at this time $x\left(t_{1}\right)<M$, there will be some flow $k$ whose rate $y_{k}\left(t_{1}\right)<M$.

On the other hand, by assumption, the initial value of $\left\{r_{i j}().\right\}$ is less than $2 M$. From the decreasing property of $\left\{r_{i j}().\right\}$, it follows that $r_{l k}\left(t_{1}\right)<2 M$. This, along with the fact that $y_{k}\left(t_{1}\right)<M$ implies that $y_{l}\left(t_{1}\right)<3 M$ leading to a contradiction. Therefore, we must have that all the flow rates are strictly less than $3 M$ for all time, and the required result will follow. We now formally prove this result.

Proof of Theorem 3.1: Since $\beta$ is assumed to be fixed throughout the proof, we drop the subscript in $M_{\beta}$, and simply use $M$ in this proof. Define

$$
t_{1}=\inf _{t>0}\left\{t: \max _{i \in\{1,2, \ldots, N\}}\left[y_{i}(t)\right] \geq 3 M\right\},
$$

where we include the possibility of $t_{1}$ being infinity (which would mean $\left.\max _{i}\left[y_{i}(t)\right]<3 M\right]$ for all $t>0$ ). Further, define the function $V_{i j}(t)$ as

$$
V_{i j}(t)=\sup _{t-2 d \leq s \leq t} r_{i j}^{2}(s) .
$$

We divide the proof into two steps. In the first step we show that under the condition on $\kappa d$ given by the statement of the theorem, $V_{i j}(t)<q V_{i j}(0) \exp (-\alpha t)$ for all $t<t_{1}$, for every $(i, j)$ and for some constants $q$ and $\alpha$. In the second step we show that $t_{1}=\infty$ and use this to conclude that $r_{i j}(t)$ converges to zero.

Step 1: Consider the delay-differential equation,

$\dot{r}_{i j}(t)=-\kappa p(x(t-d)+a)\left[y_{i}(t) r_{i j}(t-d)+y_{j}(t-d) r_{i j}(t)\right]$

for every pair $(i, j)$. Suppose that

$$
\begin{array}{r}
\kappa d \max _{t-2 d \leq s \leq t}\left[p(x(t-d)+a)\left(y_{i}(t)+y_{j}(t-d)\right)\right]< \\
1+\frac{y_{j}(t-d)}{y_{i}(t)}
\end{array}
$$


Since we are considering $t$ such that $t<t_{1}$, we have $0<$ $y_{i}(t)<3 M$ for all $i \in\{1,2, \ldots, N\}$. Further the average rate $x(t)<M$ by our assumption. Thus a sufficient condition for the preceding inequality to be satisfied is

$$
\kappa d[6 M p(M+a)]<1 .
$$

Since we also have $\kappa d<\beta$ for $M$ to be an eventual upper bound on $x(t)$, thus if $\kappa d$ satisfies the condition given by the statement of the theorem, and $q$ is chosen to satisfy

$$
1<\sqrt{q}<\frac{1}{6 M p(M+a) \kappa d},
$$

then by Lemma 3.2,

$$
V_{i j}(t)<q V_{i j}(0) \exp (-\alpha t) \forall t<t_{1},
$$

where $\alpha$ is a function of $q$. In this case choose $q$ such that,

$$
1<\sqrt{q}<\min \left(\frac{1}{\epsilon}, \frac{1}{6 M p(M+a) \kappa d}\right),
$$

where $\epsilon$ is such that the initial rates of the individual flows lie in $[M(1-\epsilon), M(1+\epsilon)]$. We will consider $q$ in the above range for the rest of the proof.

Step 2: We now show that $t_{1}$ as defined by (3) is not finite. We will show it by contradiction. Suppose $t_{1}<\infty$. Since the trajectories of $y_{i}(t)$ 's are continuous in $t$, we have $\max _{i}\left[y_{i}\left(t_{1}\right)\right]=3 M$. Suppose

$$
k=\arg \max _{i \in\{1,2, \ldots, N\}} y_{i}\left(t_{1}\right) .
$$

If $\kappa d$ satisfies the conditions given in the statement of the theorem, we further have from Step 1 that, $V_{k j}(t)<$ $q V_{k j}(0) \exp (-\alpha t)$ for all $t<t_{1}$. Since, $V_{k j}(0)<(2 M \epsilon)^{2}$ for all $j$ from our assumption on the initial condition, we have $V_{k j}(t)<q(2 M \epsilon)^{2} \exp (-\alpha t)$ for all $t<t_{1}$. From the continuity of $V_{k j}(t)$ in $t$,

$$
V_{k j}\left(t_{1}\right)<q(2 M \epsilon)^{2}
$$

which in turn implies

$$
\sup _{t_{1}-2 d \leq s \leq t_{1}}\left|r_{k j}\left(t_{1}\right)\right|<2 M \epsilon \sqrt{q}
$$

which further implies

$$
\left|y_{k}\left(t_{1}\right)-y_{j}\left(t_{1}\right)\right|<2 M \epsilon \sqrt{q} \forall j .
$$

We also have

$$
\frac{1}{N} \sum_{i=1}^{N} y_{i}\left(t_{1}\right)<M
$$

since $x(t)<M$ for all $t>0$. If the average of $N$ quantities is less than $M$, there must be at least one of them less than $M$. Let that element be indexed by $l$ so that $y_{l}\left(t_{1}\right)<M$. Note that $l \neq k$ since $y_{k}\left(t_{1}\right)=3 M$. We thus have,

$$
y_{k}\left(t_{1}\right) \leq\left|y_{k}\left(t_{1}\right)-y_{l}\left(t_{1}\right)\right|+y_{l}\left(t_{1}\right)<2 M \sqrt{q} \epsilon+M<3 M,
$$

where we have used the fact that $\sqrt{q} \epsilon<1$. But, $y_{k}\left(t_{1}\right)=3 M$. Thus we have arrived at a contradiction and so for all $t>0$, $y_{i}(t)<3 M$ for all $i \in\{1,2, \ldots, N\}$. This along with Step 1 implies that

$$
V_{i j}(t)<q V_{i j}(0) \exp (-\alpha t) \quad \forall t>0 .
$$

This proves the convergence of $r_{i j}(t)$. To show that the convergence is uniform in all pairs $(i, j)$ we simply note that the exponent in the exponential convergence only depends on the choice of $\kappa$ and not on any specific flow.

Thus, we have shown that the trajectories of all the flows get coupled if their round-trip delays are the same. Using this we can now study the stability with multiple flows by using stability results from the single flow case. First note that the average flow rate through the link $x(t)$ can be written as

$$
\begin{aligned}
x(t)= & \frac{1}{N} \sum_{i=1}^{N} y_{i}(t) \\
& =y_{k}(t)+\frac{1}{N} \sum_{i=1}^{N}\left[y_{i}(t)-y_{k}(t)\right] \\
& =y_{k}(t)+\delta(t),
\end{aligned}
$$

where $y_{k}(t)$ is any particular flow and $\delta(t)$ is a term which goes to zero exponentially. Next we rewrite the update equation for flow $k$ as follows.

$$
\begin{aligned}
\dot{y}_{k} & \\
= & \kappa\left[w-y_{k}(t) y_{k}(t-d) p\left(y_{k}(t-d)+a+\delta(t-d)\right)\right] \\
= & \kappa\left[w-y_{k}(t) y_{k}(t-d) p\left(y_{k}(t-d)+a\right)\right]+ \\
& \kappa\left[y_{k}(t) y_{k}(t-d) p\left(y_{k}(t-d)+a\right)-\right. \\
& \left.y_{k}(t) y_{k}(t-d) p\left(y_{k}(t-d)+a+\delta(t-d)\right)\right] \\
= & \kappa\left[w-y_{k}(t) y_{k}(t-d) p\left(y_{k}(t-d)+a\right)\right]- \\
& y_{k}(t) y_{k}(t-d) p^{\prime}\left(\beta_{k}(t)+a\right) \delta(t-d) \\
\Rightarrow & \dot{y}_{k}=\kappa[w- \\
& \left.y_{k}(t) y_{k}(t-d) p\left(y_{k}(t-d)+a\right)\right]+\eta(t)
\end{aligned}
$$

The second last step follows from the mean-value theorem and $\left.\beta_{k}(t)=y_{k}(t-d)+f \delta(t-d)\right)$ for some $f$ such that $0<f<1$. Note that since $|\delta(t)| \rightarrow 0$ exponentially and all the other terms are bounded, $|\eta(t)| \rightarrow 0$ exponentially. Thus we can view the trajectory of the $k$-th flow as a single flow accessing the link except for an additional term which is negligible for large $t$. It is thus natural to believe that the stability criterion for

$$
\dot{u}=\kappa[w-u(t) u(t-d) p(u(t-d)+a)]
$$

is sufficient to guarantee the stability of the system with multiple flows. We show this in the next theorem, which is a simple extension of the global stability result with single flow in [3].

Theorem 3.2: Suppose

$$
\kappa d<\min \left[\beta, R, \frac{1}{6 M_{\beta} p\left(M_{\beta}+a\right)}\right],
$$

where $R>0$ is such that $\kappa d<R$ is a sufficient condition for (6) to be globally stable. Then the system described by (5) is globally asymptotically stable. 
Before we prove the above result we state a useful result on functional differential equation [9].

Lemma 3.3 (page 79, [9]): Consider the retarded functional differential equation

$$
\dot{x}(t)=f\left(x_{t}\right), x_{0}=\phi
$$

where $x_{t}=\{x(t+\theta):-d \leq \theta \leq 0\} \in C B[-d, 0]$ and $\phi \in C B[-d, 0]$. Assume $f: C B[-d, 0] \rightarrow R^{n}$ is continuous, Lipschitz, and, $f(0)=0$. Then (7) is exponentially stable if and only if there exists a functional $V(t, \phi)$ such that

$$
\begin{array}{r}
c_{1}\|\phi\| \quad \leq V(t, \phi) \leq c_{2}\|\phi\| \\
\dot{V} \leq-c_{3}\left\|x_{t}\right\| \leq-\frac{c_{3}}{c_{2}} V, \\
|V(t, \phi)-V(t, \xi)| \leq c_{4}\|\phi-\xi\|,
\end{array}
$$

where the norms of the functions are defined as $\|\phi\|=\sup _{-d \leq \theta \leq 0}|\phi(\theta)-\xi(\theta)|$, and, $c_{i}$ are some positive constants.

Proof of Theorem 3.2: Since,

$$
\dot{y}_{k}=\kappa\left[w-y_{k}(t) y_{k}(t-d) p\left(y_{k}(t-d)+a\right)\right]
$$

is globally exponentially stable [3], we have from Lemma 3.3, the existence of a Lyapunov function $V_{k}\left(t, y_{k t}\right)$ such that

$$
\dot{V}_{k} \leq-\gamma V_{k}
$$

and satisfying the properties given in Lemma 3.3. Now, apply the Lyapunov functional

$$
V(t) \equiv V\left(t, y_{t}\right)=\frac{1}{N} \sum_{k=1}^{N} V_{k}\left(t, y_{k t}\right)
$$

to the system given by(5). Since

$$
\sup _{k}\left|\eta_{k}(t)\right| \leq K_{1} \exp (-\alpha t),
$$

it is easy to see that

$$
\dot{V} \leq-\gamma V+K_{1} c_{4} \exp (-\alpha t)
$$

from which it follows that

$$
V(t) \leq V(0) \exp (-\gamma t)-\frac{K_{1} c_{4}}{\gamma-\alpha}[\exp (-\gamma t)-\exp (-\alpha t)] .
$$

The result thus follows since all the initial conditions are assumed to lie in a compact set. We note that the exponent in the exponential stability can be chosen as $\min (\gamma, \alpha)$.

We next extend the results to the case when the noise is modeled as a time-varying process but satisfies a strong law of large number type property as discussed in Section II.

\section{Multiple TCP-LiKe Flows With Time-VARYing NOISE}

We now consider a variation of the earlier system. We consider a sequence of systems so that in the $N$-th system, the rate adaptation of the $i$-th flow is described by,

$\dot{y}_{i}^{(N)}=\kappa[w-$

$$
\begin{gathered}
\left.y_{i}^{(N)}(t) y_{i}^{(N)}(t-d) p\left(x^{(N)}(t-d)+a+e^{(N)}(t-d / 2)\right)\right], \\
i \in\{1,2, \ldots, N\},
\end{gathered}
$$

where $\left(e^{(N)}(t)+a\right)$ is the average noise due to $N$ uncontrolled flows and $x^{(N)}(t)$ is the average flow rate of $N$ TCP-like flows. Let us assume

$$
\lim _{N \rightarrow \infty} \sup _{t \in[0, N T]} e^{(N)}(t)=0 \text { a.s. , }
$$

where $e^{(N)}(t)$ is the average noise due to $N$ uncontrolled flows. Conditions for (9) to hold are given in [16]. Before we extend our results to stochastic and bounded noise process satisfying (9), in Subsection IV-A, we first study a system with the noise process satisfying a slightly relaxed assumption as follows.

$$
\lim _{N \rightarrow \infty} \sup _{t \in[0, \infty)}\left|e^{(N)}(t)\right|=0 .
$$

The case with $e^{(N)}(t)$ satisfying (9) is discussed in Subsection IV-B and the results follow from those derived in Subsection IV-A.

A. TCP-like flows with noise process satisfying SLLN type property over infinite time

We assume that,

$$
\lim _{N \rightarrow \infty} \sup _{t \in[0, \infty)}\left|e^{(N)}(t)\right|=0 .
$$

Note that the value of $\kappa$ does not change with the scaling $N$. We also have an upper bound, $M$, on the average rate when the disturbance due to uncontrolled flows is simply assumed to be a constant rate $a$. Since the average disturbance process becomes arbitrarily small as $N$ grows, the following Lemma is easy to show using the upper bound on the average rate proved in [15].

Lemma 4.1: Suppose $\kappa d<\beta$. Then given $\epsilon^{\prime}>0$, there exists $\bar{N}$ and $\bar{t}\left(\epsilon^{\prime}\right)$ such that $\forall(N \geq \bar{N})$ and $\forall(t \geq \bar{t})$,

$$
x^{(N)}(t) \leq M_{\beta}+\epsilon^{\prime},
$$

where $M_{\beta}$ is as given in Lemma 3.1

As in the previous section, define

$$
r_{i j}^{(N)}(t)=y_{i}^{(N)}(t)-y_{j}^{(N)}(t), \quad(i, j) \in\{1,2, \ldots, N\}^{2}
$$

As before, it can be shown that

$$
\begin{aligned}
\dot{r}_{i j}^{(N)}(t)= & -\kappa p\left(x(t-d)+a+e^{(N)}(t-d / 2)\right) \\
& {\left[y_{i}^{(N)}(t) r_{i j}^{(N)}(t-d)+y_{j}^{(N)}(t-d) r_{i j}^{(N)}(t)\right] . }
\end{aligned}
$$

Since the additional noise term becomes arbitrarily small for large $N$, we can expect $\left\{r_{i j}^{(N)}\right\}$ to become small for conditions 
when $\kappa d$ given by Theorem 3.1 hold. We remind the reader that for all $N$, the initial conditions are assumed to satisfy the conditions given in Assumption 2.

Theorem 4.1: If

$$
\kappa d<\min \left[\beta, \frac{1}{6 M_{\beta} p\left(M_{\beta}+a\right)}\right],
$$

then, given $\epsilon^{\prime}>0, \exists\left(\bar{t}\left(\epsilon^{\prime}\right), \bar{N}\right)$ such that $\forall(N>\bar{N})$,

$$
\sup _{t \in[\bar{t}, \infty)}\left|r_{i j}^{(N)}(t)\right|<\epsilon^{\prime}
$$

Further, if $\kappa d$ satisfies the condition given in Theorem 3.2, then, given $\epsilon^{\prime}>0, \exists N^{\prime}\left(\epsilon^{\prime}\right)$ such that $\forall\left(N>N^{\prime}\right)$,

$$
\lim _{t \rightarrow \infty}\left|y_{k}(t)-y^{*}\right| \leq \epsilon^{\prime}, \quad k \in\{1,2, \ldots N\},
$$

where $y^{*}$ is the solution of $w=y^{2} p(y+a)$.

Proof: We refer the reader to [14] for details of the proof.

\section{B. TCP-like flows with noise process satisfying SLLN type property over finite time}

If the noise process is stochastic and bounded, then it can be shown [16] that,

$$
\lim _{N \rightarrow \infty} \sup _{t \in[0, N T]} e^{(N)}(t)=0 \quad \text { a.s. },
$$

where $e^{(N)}(t)$ is the average noise due to $N$ uncontrolled flows. It is thus natural to believe that the results in the previous subsection hold for almost all realizations of the noise process satisfying the above condition.

Theorem 4.2: Suppose $e^{(N)}(t)$ satisfies (13). If $\kappa d$ satisfies the condition given in Theorem 3.2, then, given $\epsilon^{\prime}>0$, $\exists N^{\prime}\left(\epsilon^{\prime}\right)$ such that $\forall\left(N>N^{\prime}\right)$,

$$
\left|y_{k}^{(N)}(N T)-y^{*}\right| \leq \epsilon^{\prime}, \quad k \in\{1,2, \ldots N\} \quad \text { a.s. },
$$

where $y^{*}$ is the solution of $w=y^{2} p(y)$.

Proof: See [14] for the proof.

Finally, we note that in practice, the sources may update their rates at discrete time slots. We can view each time slot as a measurement interval over which rates are measured in the system and control actions by the routers and flows are updated. Typically, this measurement interval is measured in terms of the number of packets that can be processed by a typical router. For example, the time-step could be "100 packets long." By scaling both the time-step and the capacity, we maintain a constant time-step, as measured in packets [16].

To this end, let each time time-step in the $N$-th system be $1 / N$. Thus, the update of the $i$-th system at the $(k+1)$-th time-step can be described by the following.

$$
\begin{aligned}
& y_{i}^{(N)}[k+1]=y_{i}^{(N)}[k]+ \\
& \quad \frac{\kappa}{N}\left[w-y_{i}^{(N)}[k] y_{i}^{(N)}[k-N d] p\left(x^{(N)}[k-N d]+\right.\right. \\
& \left.\left.\quad e^{(N)}[k-N d / 2]+a\right)\right]
\end{aligned}
$$

Note that, since the delay $d$ as measured in seconds is fixed, the delay in the $N$-th system corresponds to $N d$ time slots. The continuous time model can now be embedded as

$$
y_{i}^{(N)}(t)=y_{i}^{(N)}[N t], \text { for } N t \in \mathbb{N}
$$

with a straight line approximation used between integers. Similarly, the average rate process $x^{(N)}(t)$ and the noise process $e^{(N)}(t)$ can be defined. For such a system of the continuous time processes $y_{i}^{(N)}(t)$, the results in Theorem 4.2 can be proved as well using techniques analogous to those used in [16]. For brevity, we skip the details here.

\section{Stability Conditions With STANDARd TCP}

In previous sections, we have seen that that stability condition with a single TCP connection accessing a link along with some additional condition is enough to ensure the stability and the convergence of multiple TCP-like flows in a many flows regime.

In this section, we use the earlier results to derive stability conditions with the standard TCP parameters. Since the global stability criterion of a single TCP-flow accessing a bottleneck link plays an important role in the many flows regime, we first state the stability condition with a single TCP flow with parameters implied by standard TCP. Recall that the congestion avoidance phase of TCP can be modeled as [10],

$$
\dot{x}(t)=\frac{1}{d^{2}}-\frac{2}{3} x(t) x(t-d) p(x(t-d)+a, c),
$$

where $x$ is in segments (each segment may correspond to 512 bytes) per time unit and $a$ is the mean flow rate due to the uncontrolled flows. We define $w(t)$ as

$$
w(t)=d x(t)
$$

The evolution of the congestion-avoidance phase of TCP can be re-written as,

$$
\dot{w}(t)=\frac{2}{3 d}\left[\frac{3}{2}-w(t) w(t-d) p\left(\frac{w(t-d)+a d}{c d}, 1\right)\right] .
$$

Since $x(t)$ is the rate in segments per unit time and $d$ is the round trip time, $w(t)$ can be interpreted as the congestion window size in segments. Further, the quantity $c d$ determines the desired bandwidth-delay product per source at the equilibrium. Thus, (15) is a continuous time version of the window update algorithm of TCP algorithm in the congestion avoidance phase.

The following lemma provides sufficient conditions under which (15) is globally exponentially stable. The condition follows from the results derived in [3]. We state the stability condition of (15) below, which follows from Theorem 2.2 in [3]. For the the rest of this section, we let

$$
q(w)=p\left(\frac{w}{c d}, 1\right) .
$$

Lemma 5.1: The controller given by (15) is globally exponentially stable if,

$$
\frac{2}{3}<\frac{l^{2}\left(\frac{3}{2}+M^{2} R\right)}{M^{3} R\left(\frac{3}{2}+l^{2} R\right)},
$$


where, $R=\sup _{l<w<M}\left(q(w)+w q^{\prime}(w)\right), M$ is the smallest positive number satisfying

$$
M(M-1) p(M-1+a d) \geq \frac{3}{2},
$$

and $l$ is the largest positive number satisfying

$$
\begin{array}{r}
l\left(l+\frac{2}{3} M^{2} q(M+a d)-1\right) \times \\
q\left(l+\frac{2}{3} M^{2} q(M+a d)-1+a d\right) \leq \frac{3}{2}
\end{array}
$$

Now we consider multiple TCP-flows. We further consider only the mean flow rate of the uncontrolled flows, since the stability of such a system is sufficient to ensure the stability of the system with stochastic disturbances when the number of flows is large. Continuing with the earlier model, let there be $N$ flows, with the update equation of the $i$-th flow described by

$$
\begin{gathered}
\dot{w}_{i}(t)=\frac{2}{3 d}\left[\frac{3}{2}-w_{i}(t) w_{i}(t-d) q(w(t-d)+a d)\right] \\
i \in\{1,2, \ldots N\},
\end{gathered}
$$

where $w(t)$ is the average window size $(w(t)=d x(t)$ where $x(t)$ is the average flow rate) of the $N$ flows. Using Theorem 3.2, we can easily derive stability conditions for the system described by (16). The following result provides such conditions.

Corollary 5.1: The system given by (16) is globally stable if the following conditions hold:

1) We have $\frac{2}{3} M_{1} q\left(M_{1}+a\right)<\frac{1}{6}$, where $M_{1}$ is the smallest positive number satisfying

$$
\left(M_{1}-1\right)^{2} q\left(M_{1}+a d-2\right)\left(1-\frac{1}{M_{1}-1}\right) \geq \frac{3}{2} .
$$

2) The condition given by Lemma 5.1 is satisfied.

We next present examples for two different marking functions to demonstrate the usefulness of the previous result. We are interested in finding the range of the equilibrium bandwidth-delay product to ensure stability and convergence of multiple TCP flows. We now demonstrate that, for the examples considered, we can ensure global stability if the bandwidth-delay product is large enough.

So far we have assumed that the marking function is a function of the total flow rate through the link. However, note that one could also consider queue based marking at the router (such as RED or REM). Given a queue based marking function, using a reflected Brownian motion (RBM) approximation, a technique to find an equivalent rate based marking function was suggested in [8]. We consider two marking functions in the following examples: first, a rate based marking function and second, a rate based marking function equivalent to REM, which is a queue based marking mechanism.

Example $1(M / M / 1$ type marking function):
We consider the marking function,

$$
p(x, c)=\left(\frac{x}{c}\right)^{B}=\left(\frac{w}{c d}\right)^{B} .
$$

Here $x$ is the average flow rate of the sources through the link and $c$ is a parameter which can be adjusted for a desired bandwidth per source at the equilibrium. Such a marking function has the interpretation of probability of the buffer size being larger than $B$ in an $M / M / 1$ queue with arrival rate $x$. The equilibrium rate per source $x^{*}$ in this case is given by

$$
x^{*} d=\left(\frac{3}{2}\right)^{\frac{1}{B+2}}(c d)^{\frac{B}{B+2}} .
$$

We are interested in finding values of equilibrium bandwidthdelay product $x^{*} d$ to guarantee global stability of multiple TCP flows.

We show that the conditions given by Corollary (5.1) are satisfied as $x^{*} d \rightarrow \infty$. To make our calculations easy, we assume that $(c d)^{B /(B+2)}>5$ for reasons which will become obvious soon. In other words, we seek values of $x^{*} d$ in the range $\left[5(3 / 2)^{1 /(B+2)}, \infty\right)$ to ensure global stability of multiple identical TCP-like flows. For the given marking function, first note that we have for $M$ given in Lemma 5.1

$$
\begin{aligned}
M & <1+(c d)^{\frac{B}{B+2}}(3 / 2)^{\frac{1}{B+2}} \\
& <(c d)^{\frac{B}{B+2}}\left[\frac{1}{5}+\left(\frac{3}{2}\right)^{\frac{1}{B+2}}\right]=K(c d)^{\frac{B}{B+2}},
\end{aligned}
$$

where

$$
K=\frac{1}{5}+\left(\frac{3}{2}\right)^{\frac{1}{B+2}} .
$$

It can also be easily seen that

$$
M^{2} q(M)<K^{B+2} .
$$

Also, for $l$ given in Lemma 5.1, we have

$$
\begin{aligned}
l & >1+(c d)^{\frac{B}{B+2}}(3 / 2)^{\frac{1}{B+2}}-\frac{2}{3} M^{2} p(M) \\
& >(c d)^{\frac{B}{B+2}}\left[\left(\frac{3}{2}\right)^{\frac{1}{B+2}}-\frac{1}{5}\left(\frac{2}{3} K^{B+2}-1\right)\right] .
\end{aligned}
$$

It follows from some algebraic manipulations that Condition 2 in Corollary 5.1 is satisfied when

$$
M\left(\frac{3}{2 l^{2}}+R\right)<\frac{3}{2},
$$

which is satisfied if

$$
(c d)^{\frac{B}{B+2}} \geq \frac{2}{3} K^{B+1}(1+B)+\frac{K}{\left(\left(\frac{3}{2}\right)^{\frac{1}{B+2}}-\frac{1}{5}\left(1-\frac{2}{3} K^{B+2}\right)\right)^{2}} .
$$

Next note that $M_{1}$, the upper bound on the average rate in condition 1 of Corollary 5.1, is such that

$$
M_{1}<\left(\frac{3}{2}\right)^{\frac{1}{B+2}}(c d)^{\frac{B}{B+2}}+2<K_{1}(c d)^{\frac{B}{B+2}},
$$


where

$$
K_{1}=\frac{2}{5}+\left(\frac{3}{2}\right)^{\frac{1}{B+2}} .
$$

One can check that a sufficient condition for Condition 1 in Corollary 5.1 to be satisfied is

$$
(c d)^{\frac{B}{B+2}} \geq 4 K_{1}^{B+1} .
$$

Since $x^{*} d=(3 / 2)^{1 /(B+2)}(c d)^{b /(B+2)}$, a sufficient global stability criterion for multiple TCP-like flows is

$$
\begin{aligned}
x^{*} d \geq & \left(\frac{3}{2}\right)^{\frac{1}{B+2}} \max \left[\frac{2}{3} K^{B+1}(1+B)+\right. \\
& \left.\frac{K}{\left(\left(\frac{3}{2}\right)^{\frac{1}{B+2}}-\frac{1}{5}\left(\frac{2}{3} K^{B+2}-1\right)\right)^{2}}, 4 K_{1}^{B+1}, 5\right] .
\end{aligned}
$$

It follows that the system is globally stable as $x^{*} d \rightarrow \infty$.

With a more accurate numerical calculation based on Corollary 5.1, it can be verified that for $B=8$, the system is globally stable for

$$
x^{*} d \geq 16.66
$$

which corresponds to at least 8 packets per source at the equilibrium since each packet approximately consists of $2 \mathrm{seg}$ ments. For $B=5$ a sufficient condition is,

$$
x^{*} d \geq 13.43
$$

corresponding to at least 7 packets per source at the equilibrium with packet sizes of 2 segments.

One can obtain similar results for the marking function

$$
p(x)=\frac{\left(\frac{x}{c}\right)^{B}\left(1-\left(\frac{x}{c}\right)\right)}{1-\left(\frac{x}{c}\right)^{B+1}},
$$

which can viewed as the blocking probability in an $M / M / 1 / B$ queue with arrival rate $x$ and service rate $c$. A sufficient condition with $B=8$ is

$$
x^{*} d \geq 14.97 \text {, }
$$

and, with $B=5$,

$$
x^{*} d \geq 12.44
$$

provides a sufficient condition for global stability.

\section{Example 2 (Random Early Marking or REM):}

REM [1] marks a packet with probability $(1-\exp (-\theta W))$ if it arrives to find a workload of $W$ already present in the virtual queue. Using a reflected Brownian motion approximation [8] this can be viewed as a mechanism with the following marking function:

$$
p(x, c)=\frac{\theta \sigma^{2} x}{\theta \sigma^{2} x+2(c-x)}
$$

Here, $\sigma^{2}$ denotes the variability of the traffic and $c$ can be tuned to obtain a desired rate allocation at the equilibrium.
For simplicity, we assume $\theta \sigma^{2}=0.5$. The equilibrium rate allocation can be obtained by solving

$$
\frac{0.5\left(x^{*} d\right)^{3}}{0.5 x^{*} d+2\left(c d-x^{*} d\right)}=\frac{3}{2} .
$$

It can be verified that

$$
x^{*} d \leq(6 c d)^{\frac{1}{3}} .
$$

First we argue that the global stability condition is satisfied as $x^{*} d \rightarrow \infty$. Suppose we consider $c d$ in the range $c d \geq 5$, which corresponds to $x^{*} d \geq 2.63$. We are interested in finding $x^{*} d$ in the range $[2.37, \infty)$ to guarantee stability. Note that the parameter $M$ in Lemma 5.1 satisfies

$$
K_{1}(c d)^{\frac{1}{3}} \leq M \leq K_{2}(c d)^{\frac{1}{3}},
$$

for suitable constants $K_{1}$ and $K_{2}$. Further, it can be shown that

$$
l \geq K_{3}(c d)^{\frac{1}{3}}
$$

and

$$
\left.R=\sup _{l \leq w \leq M} q(w)+w q^{\prime}(w)\right) \leq K_{4}(c d)^{-\frac{2}{3}}
$$

for appropriate positive constants $K_{3}$ and $K_{4}$. It follows that Condition 2 in Corollary 5.1 is satisfied when

$$
M\left(\frac{3}{2 l^{2}}+R\right)<\frac{3}{2},
$$

which is satisfied if

$$
(c d)^{\frac{1}{3}} \geq \frac{2}{3} K_{2}\left(\frac{1}{K_{3}}+K_{4}\right) .
$$

Similarly, condition 1 is Corollary 5.1 can be expressed as

$$
(c d)^{\frac{1}{3}} \geq K_{5} .
$$

Since $x^{*} d \leq(6 c d)^{\frac{1}{3}}$, a sufficient condition for global stability in this case is

$$
x^{*} d \geq K
$$

for a suitable constant $K$.

One can use numerical calculations to obtain the a sufficient condition for global stability with multiple TCP flows as

$$
x^{*} d \geq 8.67 \text {. }
$$

This corresponds to at least 5 packets per source at the equilibrium with packet sizes of 2 segments.

The above examples clearly indicate that:

For reasonable marking functions and large enough target bandwidth-delay product per source, multiple TCP flows eventually behave like a single flow and the system is globally asymptotically stable. 


\section{Simulation Results}

In this section we present simulation results to illustrate the effectiveness of deterministic fluid models in designing marking function to meet a target equilibrium rate allocation. The purpose of the simulation is two-fold. First, we show that if the target equilibrium window size or the bandwidth-delay product is large (in the range predicted by the global stability criterion), then, the equilibrium window size in a packet based simulation is close to the one predicted by the deterministic fluid models.

Second, in our earlier sections we have derived condition for global stability of a system with TCP-like flows. The natural question is: Why is stability so important? To illustrate the importance of designing parameters to ensure stability, we compare the performance of TCP with a modified version. We show that large oscillations can happen due to instability in a badly designed system.

We simulate a single bottleneck link being accessed by 100 persistent flows. All the long-lived flows are TCP-flows using the dynamic window flow control mechanism of TCP. The simulation is packet based and the link marks a fraction of the packets in the event of a congestion and the marks are echoed back to the source. There are also uncontrolled flows (around $20 \%$ of the link bandwidth), i.e., flows that do not react to congestion. The link has an $M / M / 1$ type marking function given by the following.

$$
p(\hat{x})=\left(\frac{\hat{x}}{\tilde{C}}\right)^{B} .
$$

Here $\hat{x}$ is the estimated arrival rate at the link and $\tilde{C}$ is the virtual capacity. Using the fluid deterministic model, the value of $\tilde{C}$ required to meet a target per source equilibrium rate of $y^{*}$ is given by

$$
\tilde{C}=N\left(\frac{3}{2}\left(y^{*}+\frac{a}{N}\right)^{B+2} d^{2}\right)^{\frac{1}{B}}
$$

where $d$ is the round trip time, $N$ is the number of flows, and, $a$ is the mean flow rate due to the unresponsive flows. In our simulations, we choose $N=100, d=50 \mathrm{~ms}$, and, $a=20 \%$ of the link bandwidth. The parameter $B$ in the marking function is chosen as $B=8$. For the purposes of our simulation we simply simulate the congestion avoidance phase of TCP so that each successful transmission increases the current window size $W$ by $1 /\lfloor W\rfloor$ and each mark reduces the window size by half. We also note that such a description of the window update assumes the window size to be in segments. In the following we assume that each segment is equivalent to one packet.

We first choose $\tilde{C}$ to ensure total equilibrium rate of 40.5 packets $/ \mathrm{ms}$, which is equivalent to an equilibrium window size per flow of roughly 16 packets. The plots comparing the total rate into the link and the target rate are shown in Figure 3. The plot is representative of simulations done with different choices of parameters and for a large number of flows. The simulation seems to indicate that, for moderately large target equilibrium window size (in the range predicted by the stability criterion), deterministic fluid models based parameter and marking function design is appropriate.

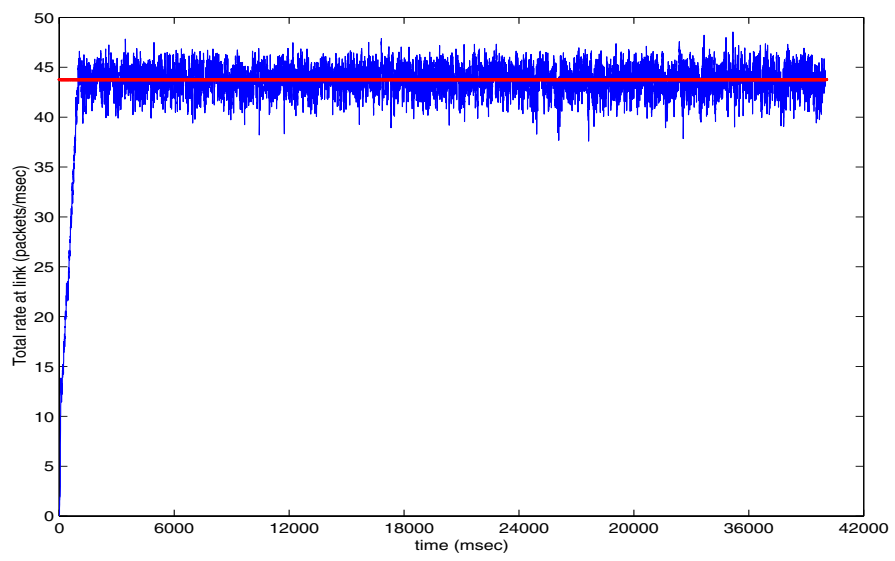

Fig. 3. Plot showing evolution of average rate (averaged over one round trip time) of a typical flow and the rate predicted by the fluid model
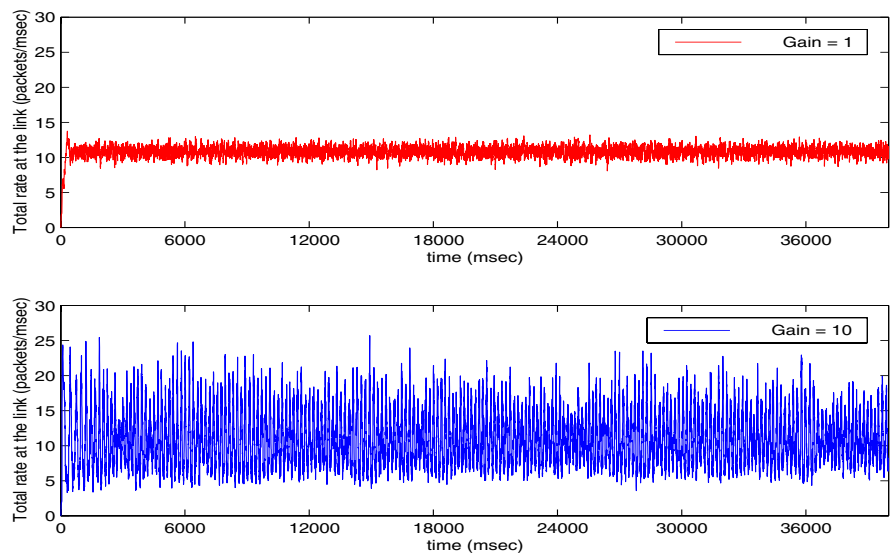

Fig. 4. Plot showing evolution of average rate (averaged over one round trip time) of a typical flow and the rate predicted by the fluid model for two cases: the standard TCP and a modified unstable TCP

We next demonstrate the oscillations that can happen in a badly designed system. We consider a modified version of TCP in which each successful transmission increases the window size $W$ by $10 /\lfloor W\rfloor$, and, each marked packet reduces the window size by half as in standard TCP. In Figure 4, we compare the average rate of this modified TCP with that of a standard one for a target equilibrium rate of 8.33 packets $/ \mathrm{ms}$. Clearly, the modified version of TCP has larger fluctuations in the average rate. This may even lead to a lower throughput in a drop based system (where packets are dropped at congestion instead of being marked) with re-transmission of dropped packets. The variance of total rate at the router in the case of this modified TCP is about 16 , whereas the variance is 0.63 for the case of standard TCP. This underlines the importance of ensuring stability. 


\section{CONCLUSION}

We have studied a system consisting of a single link accessed by a large number of TCP-like flows, each with identical delay access, but with (possibly) different initial condition and also accessed by a large number of uncontrolled flows. The contributions of this paper are:

(i) Our main result is that in the presence of uncontrolled flows (stochastic noise), if the number of flows is large enough, the global exponential stability criterion for a single flow (with minor modifications) is also a global exponential stability condition for the stochastic system with multiple flows. Thus, the implication is that parameter design can be carried out using deterministic analysis based on the single flow model.

(ii) For the rate adaptation model of TCP [10], we have shown that the stability is ensured if the target equilibrium delay-bandwidth product (window size) per flow is large enough, and we have derived upper bounds on this quantity. Thus, we have derived sufficient conditions for global stability. Numerical examples with two popular marking functions indicate that the target window size per flow required to ensure stability is actually not very large.

(iii) Using simulations, we have verified that the deterministic fluid models that are stable can be used to design active queue management mechanisms.

\section{ACKNOWLEDGMENT}

The authors would like to acknowledge that the research was supported by AFOSR URI F49620-01-1-0365 and DARPA Grant F30602-00-2-0542.

\section{REFERENCES}

[1] S. Athuraliya, D. E. Lapsley and S. H. Low, Random early marking for Internet congestion control, Proceedings of IEEE GLOBECOM, 1999.

[2] F. Baccelli and D. Hong, Interaction of TCP Flows as Billiards, Technical Report, April 2002, INRIA Rocquencourt

[3] S. Deb and R. Srikant, Global Stability of Congestion Controllers for the Internet, Proceedings of IEEE Conference on Decision and Control, Las Vegas, Dec, 2002.

[4] S. Floyd, TCP and explicit congestion notification, ACM Computer Communication Review, 24:10-23, October, 1994.

[5] C.V. Hollot, V. Misra, D. Towsley and W. Gong, On designing improved controllers for AQM routers supporting TCP flows, Proceedings of INFOCOM 2001, Anchorage, Alaska, April, 2001.

[6] R. Johari and D. Tan, End-to-End Congestion Control for the Internet: Delays and Stability, IEEE/ACM Transactions on Networking, 9(6):818832, December, 2001.

[7] F. P. Kelly, A. Maulloo and D. Tan, Rate control in communication networks: shadow prices, proportional fairness and stability, Journal of the Operational Research Society, 49:237-252, 1998.

[8] F.P. Kelly, Models for a self-managed Internet, Philosophical Transactions of the Royal Society, A358:2335-2348, 2000.

[9] V. B. Kolmanovskii and V. R. Nosov, Stability of Functional Differential Equations, Academic Press, INC, Orlando, Florida, USA, 1986.

[10] S. Kunniyur and R. Srikant, End-to-end congestion control: utility functions, random losses and ECN marks, Proceedings of INFOCOM 2000, Tel Aviv, Israel, March, 2000.

[11] S. Kunniyur and R. Srikant, Analysis and design of an adaptive virtual queue algorithm for active queue management, Proceedings of ACM Sigcomm, San Diego, CA, August, 2001.
[12] L. Massoulie, Stability of distributed congestion control with heterogenous feedback delays, IEEE Transactions on Automatic Control, 47(6):895-902, June, 2002.

[13] F. Paganini, J. Doyle and S. Low, Scalable Laws for Stable Network Congestion Control, Proceedings of the IEEE Conference on Decision and Control, Dec, 2001

[14] S. Deb, S. Shakkottai and R. Srikant, Stability and Convergence of TCP Flows in a Many-flows regime, Technical Report, Coordinated Science Lab, UIUC, Urbana, IL", 2002

[15] S. Shakkottai, R. Srikant and S. Meyn, Bounds on the Throughput of Congestion Controllers in the Presence of Feedback Delay, Proceedings of the IEEE Conference on Decision and Control, Florida, Dec, 2001

[16] S. Shakkottai and R. Srikant, Mean FDE models for Internet Congestion Control, Technical report. A shorter version to appear in the Proceedings of IEEE INFOCOM, 2002

[17] G. Vinnicombe, On the stability of end-to-end congestion control for the Internet, University of Cambridge Technical Report, 2001 